\title{
Development of Pathological Diagnosis Support System Using Micro-computed Tomography
}

\author{
Tomonari Hayakawa ${ }^{1}$, Atsushi Teramoto ${ }^{1}$, Yuka Kiriyama², Tetsuya Tsukamoto ${ }^{2}$, \\ Ayumi Yamada ${ }^{1}$, Kuniaki Saito ${ }^{1}$ and Hiroshi Fujita ${ }^{3}$ \\ ${ }^{1}$ Graduate School of Health Sciences, Fujita Health University, 1-98 Dengakugakubo, Kutsukake, Toyoake, Aichi 470-1192, \\ Japan, ${ }^{2}$ School of Medicine, Fujita Health University, 1-98 Dengakugakubo, Kutsukake, Toyoake, Aichi 470-1192, Japan and \\ ${ }^{3}$ Faculty of Engineering, Gifu University, 1-1 Yanagido, Gifu, Gifu 501-1194, Japan
}

Received November 24, 2020; accepted March 3, 2021; published online April 22, 2021

\begin{abstract}
In pathological diagnosis, the cutting position of pathological materials is subjectively determined by pathologists. This leads to a low cutting accuracy, which in turn may lead to incorrect diagnoses. In this study, we developed a system that supports the determination of the cutting position by visualizing and analyzing the internal structure of pathological material using micro-computed tomography (CT) before cutting. This system consists of a dedicated micro-CT and cutting support software. The micro-CT system has a fixture for fixing the target, enabling the scanning of easily deformable pathological materials. In the cutting support software, a function that interactively selects the extraction plane while displaying the volume rendering image and outputs a pseudo-histological image was implemented. Our results confirmed that the pseudo-histological image showed the fine structure inside the organ and that the latter image was highly consistent with the pathological image.
\end{abstract}

Key words: pathological diagnosis, micro-computed tomography, diagnosis support

\section{Introduction}

Pathological diagnosis is carried out to evaluate the malignancy of tumors and to grade cancer; hence, it plays an important role in identifying lesions and selecting the treatment policy thereafter [16]. In pathological diagnosis, the specimen, such as a tissue or a cell directly collected from a lesion of a patient or an organ resected using surgery, undergoes macroscopic and microscopic analyses [8] via inspection methods such as cytology, histology (biopsy), surgical material diagnosis (intraoperative rapid diagnosis), and pathological dissection. Except in cytology, wherein a relatively large sample is collected, a lesion in pathological diagnosis is cut using a scalpel and then mounted onto glass slides. The specimens are then prepared by paraffin-embedding the cut blocks, thin sectioning, and

Correspondence to: Atsushi Teramoto, Graduate School of Health Sciences, Fujita Health University, 1-98 Dengakugakubo, Kutsukake, Toyoake, Aichi 470-1192, Japan. E-mail: teramoto@fujita-hu.ac.jp staining. However, as the cutting position is usually determined using only palpation and visual judgment, cutting accuracy varies substantially among pathologists. This may make identifying the tissue lesion difficult, as the lesion to be diagnosed may not even be present on the slide. Eventually, this may lead to incorrect diagnosis or the use of an inappropriate treatment policy. In contrast, if the pathological material could be analyzed non-destructively and three-dimensionally, it is possible to determine its internal microstructure, and as a result, an accurate cutting position can be selected. An accurate cutting position will then in turn improve cutting accuracy.

Presently, the computed tomography (CT) scanner is widely used for non-destructive three-dimensional analysis of pathological material; furthermore, various special purpose CT scanners have been developed. In this study, we focus on the micro-CT scanner. The micro-CT scanner uses a micro-focus X-ray tube with a focal diameter of several micrometers; notably, it can visualize the three-dimensional fine internal structures of materials with a significantly 


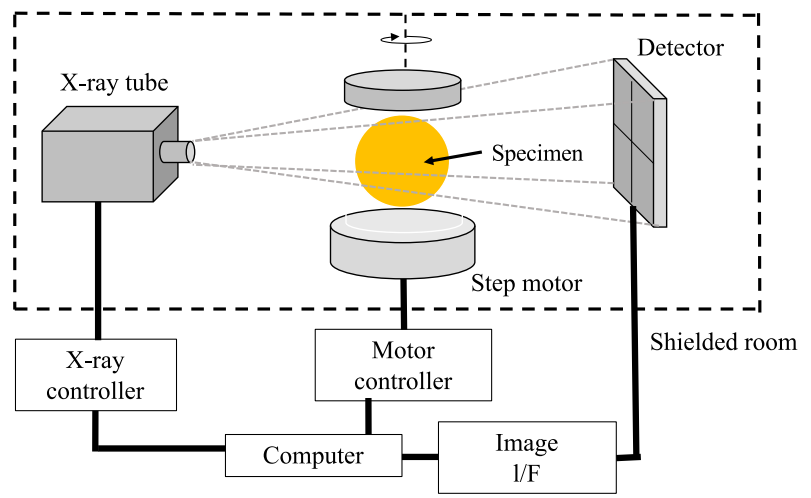

(a) Connection diagram

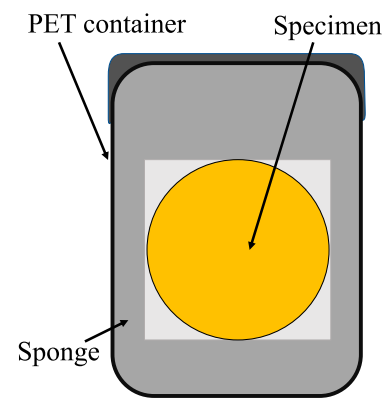

(b) Object fixing

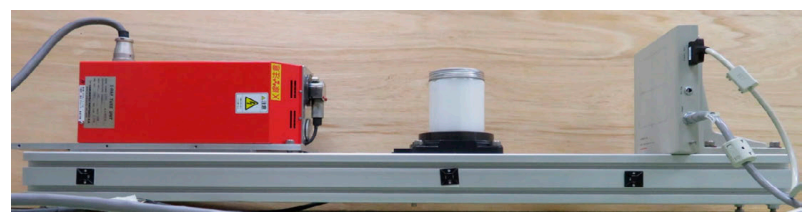

(c) External view

Fig. 1. Dedicated micro-CT system. (a) Connection diagram. (b) Object fixing. (c) External view.

higher spatial resolution than that of CT scanners [10]. Moreover, micro-CT scanners have been widely employed for investigating the pathophysiology of smaller animals, such as mice and rats, and various reports have noted them to be a useful analysis tool $[2,3,6,7,9,10]$. Therefore, a micro-CT scanner can help in non-destructively and three-dimensionally analyzing the internal microstructures of pathological material before cutting. Consequently, the exact cutting position can be determined based on the results, which can support the cutting of pathological materials. In recent years, studies using micro-CT for human organs, such as the evaluation of resected lesions in pathological diagnosis, have been performed [11-13, 15, 17]. Furthermore, several previous studies have reported the pathological effectiveness of micro-CT in evaluating surgical materials for breast cancer $[12,15]$.

Nevertheless, no dedicated system for scanning and analyzing pathological materials has been developed. To adopt micro-CT scanners for pathological diagnosis, it is necessary to develop a micro-CT system dedicated to pathological diagnosis using an object-fixing mechanism and an analysis software that supports cutting guided by acquired $\mathrm{CT}$ images.

Therefore, in this study, we develop a pathological diagnosis support system using a dedicated micro-CT scanner and analysis software.

\section{Materials and Methods}

This proposed pathological diagnosis support system consists of a dedicated micro-CT system that scans a subject and obtains a CT image, in addition to a cutting support software that analyzes the images.

\section{II.1. Overview of dedicated micro-CT system}

Fig. 1a and 1c show a connection diagram and an external view of a dedicated micro-CT scanner. It consists of a scanning unit installed inside the protective wall and its control unit. The scanning unit includes an X-ray tube, a detector, and a step motor for positioning an object. Furthermore, a micro-focus X-ray tube (L7901, Hamamatsu Photonics) with a focal diameter of $5 \mu \mathrm{m}$, maximum tube voltage of $100 \mathrm{kV}$, and maximum tube current of 100 $\mu \mathrm{A}$ was used. An indirect conversion-type flat panel detector (C7942, Hamamatsu Photonics) with a pixel pitch of $50 \mu \mathrm{m}$, phosphor of CsI, and $2366 \times 2368$ pixels was employed. Unlike conventional CT systems, this system does not rotate the X-ray tube and detector around the subject because of the small size of the object. The projection data are collected by placing the object on the center rotating stage and rotating it while scanning. The control unit is connected to a computer, an X-ray controller, a motor controller for the rotating stage, and an image interface. The computer automatically controls X-rays and motors and transfers the projection data.

The pathological specimens used in the present study, when directly placed on the rotating stage and scanned, appear soft and deformed or move during scanning, which may cause artifacts owing to the movement. To address this, we used a fixture for fixing the specimens, as shown in Fig. 1b. A PET container was used for the outer frame of the fixture, and the inside was covered with sponge with a thickness of $10 \mathrm{~mm}$. Depending on the size of the organ, three types of fixtures with different sizes were employed.

\section{II.2. Image reconstruction}

The dedicated micro-CT scanner performs cone-beam 


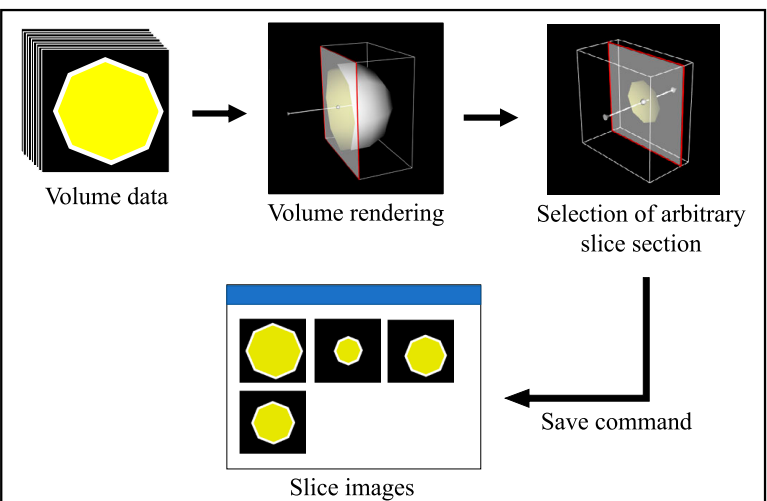

(a) Operating procedure

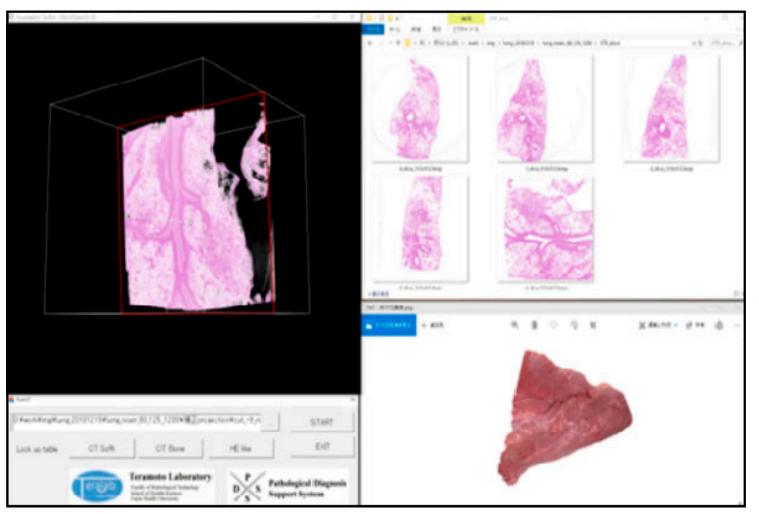

(b) Screen shot of the software

Fig. 2. Cutting support software. (a) Operating procedure. (b) Screen shot of the software.

$\mathrm{CT}$, which collects two-dimensional projection data and uses them to produce three-dimensional images. Various three-dimensional image reconstruction methods have been studied for cone-beam CT. We conducted image reconstruction using the Feldkamp-Davis-Kress (FDK) method [4, 14], which is widely used. Hereinafter, the principle of image reconstruction by the FDK method is briefly described.

\section{II.2.1. Image reconstruction processing using the FDK method}

The FDK method comprises two processes, a filtering process and a back-projection process. First, the filtering processing performs filter correction processing on the twodimensional projection data $p^{\prime}(u, v)$ in a frequency space to obtain a corrected image $P(u, v)$.

$$
P(u, v)=\frac{S I D}{\sqrt{S I D^{2}+u^{2}+v^{2}}} F^{-1}\left\{p^{\prime}(u, v) \cdot H(\omega)\right\}
$$

Here, $F^{-1}$ denotes a two-dimensional inverse Fourier transform. $H(\omega)$ is a reconstructed filter function and denotes a frequency characteristic of the filter processing. In the back-projection process, $P(u, v)$ is back-projected to a three-dimensional space $f(x, y, z)$. The voxel value at any of the aforenoted points is calculated as in Eq.(2).

$$
f(x, y, z)=\frac{1}{2 \pi N D^{2}} \cdot P(u(x, y), v(x, y))
$$

where $N$ represents the number of projections. $u(x, y)$ and $v(x, y)$ are defined as follows:

$$
\left\{\begin{array}{l}
u(x, y)=\frac{S I D-x \cos \theta-y \sin \theta}{S O D-x \cos \theta-y \sin \theta} \\
v(x, y)=\frac{S I D \cdot z}{S O D-x \cos \theta-y \sin \theta}
\end{array}\right.
$$

By performing the aforenoted processing on all the projection data obtained, a three-dimensional image $f(x, y, z)$ of the object is obtained.

\section{II.3. Outline of the cutting support software}

We developed the cutting support software to display a volume rendering (VR) image of the pathological material and to visualize and store the slice images at arbitrary angles and positions interactively. Fig. 2a shows the operation procedure of the developed software. Using the volume data acquired by the micro-CT scanner, a VR image is generated and displayed on a window. Furthermore, a slice plane associated with the VR image and a normal vector to the slice plane are also displayed on the window. By moving them using a mouse, it is possible to select an angle direction based on the normal vector, select a $\mathrm{z}$-axis direction in a three-dimensional space based on the slice plane, and select an arbitrary slice section. Furthermore, it is possible to save the selected cutting section via key operation. The supported image formats are raw, bmp, jpeg, pnm, png, and tiff. Fig. $2 b$ shows a screenshot of the software. The upper left of the figure displays a VR image window; command buttons for selecting and executing the acquired volume data are in the bottom left. The upper right shows a selected slice image (pseudo-histological image) storage file, and the lower right presents an entire photograph of the specimen. In this study, a look-up table for the slice image was set so that the color tones of the VR image and pseudo-histological image were close to that of the specimen after staining. With regard to the development environment of the cutting support software, the open source software VTK (Kitware Visualization Toolkit) [1], which excels in image processing and $3 \mathrm{D}$ data visualization, was used. The program was written in $\mathrm{C}++$ using Visual Studio 2012 (Microsoft).

\section{II.4. Evaluation methods}

To evaluate whether the proposed system can support the cutting process in pathological diagnosis, we evaluated the visualization ability for porcine organ structures. Furthermore, comparative evaluation between the pseudohistological diagnosis image and the pathological image 


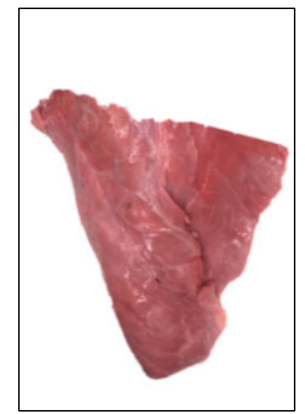

(a) Target organ (lung)

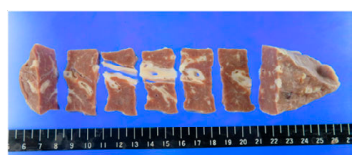

(c) Cutting cross-section

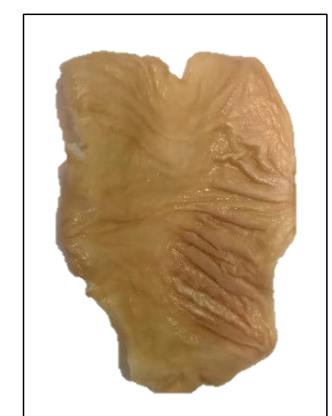

(b) Target organ (colon)

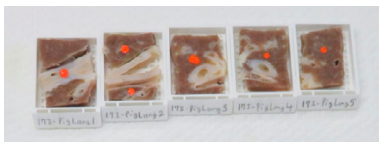

(d) Inserting into a cassette for embedding

Fig. 3. Preparation procedures of glass specimens. (a) Target organ (lung). (b) Target organ (colon). (c) Cutting cross-section. (d) Inserting into a cassette for embedding.

was performed. In this study, we focus on the porcine lung and colon as the target organs (Fig. 3a, 3b).

\section{II.4.1. Visualization ability evaluation method}

To evaluate the visualization ability of a dedicated micro-CT scanner, CT imaging was performed on the pretreated porcine organs; the obtained CT images were subjectively evaluated.

\section{(1) Sample preparation}

The Fujita Health University ethical committee for institutional animal care and use approved the use of pig cadavers from a slaughterhouse without permission.

- Formalin fixation

Formalin fixation was performed to prevent the spoilage of tissues and cells owing to autophagy and to maintain the structure of tissues and cells as close as possible to that in a living state. In this study, we fixative used was formalin (10\%), which is most widely used in the preparation of tissue specimens in clinical practice. The fixing time was 12 hours, which is the same as that of pathological material collected via surgery. The fixed organ was sealed in a zippered bag. In addition, formalin adhering to the organs was lightly removed when the images were taken.

\section{- Fixation of organs}

To prevent deformation and displacement during CT scans, and to suppress the appearance of artifacts due to movement, the specimen was fixed in the fixture as described in Section II.1 (Fig. 1b). Regarding the fixation of the colon, it was attached to an expanded polystyrene plate and inserted into the fixture since the original sponge fixture showed insufficient fixation accuracy.
Table 1. Imaging and experimental conditions

$\begin{array}{lc}\text { Organ } & \text { lung, colon } \\ \text { Tube voltage } & 80 \sim 90 \mathrm{kV} \\ \text { Tube current } & 111 \sim 125 \mu \mathrm{A} \\ \text { Number of projections } & 1200 \\ \text { Scan time } & 4.0 \mathrm{sec} / \text { projection } \\ \text { Spatial resolution } & 20.0 \mu \mathrm{m} \\ \text { Field of view size } & 4.9 \times 4.9 \mathrm{~cm} \\ \text { Voxel size } & 512 \times 512 \times 512 \\ \text { Slice thickness } & 0.1 \mathrm{~mm} \\ \text { SID } & 208 \mathrm{~mm} \\ \text { SOD } & 504 \mathrm{~mm} \\ \text { Fixative } & 12 \text { hours } \\ \text { Fixation time } & \text { Alcohols }(70 \%, 95 \%, 100 \%) \\ \text { Dehydration agent } & \text { Xylene } \\ \text { Clearing agent } & \text { Paraffin } \\ \text { Embedding agent } & 3 \mu \mathrm{m} \\ \text { Pathological slice thickness } & \text { HE } \\ \text { Staining method } & \end{array}$

SID; source to image distance, SOD; source to object distance.

(2) CT imaging with dedicated micro-CT system

Projection data were collected by scanning the preprocessed organ using a dedicated micro-CT scanner. The imaging conditions are presented in Table 1. Among these conditions, the optimal tube voltage, tube current, and number of projections vary depending on the imaging target. The conditions used in this table were determined through preliminary experiments. Subsequently, three-dimensional image reconstruction was performed on the collected projection data. In addition, a slice CT image of an arbitrary cross-section used for the creation and evaluation of the VR image was obtained through the cutting support software.

\section{II.4.2. Comparative evaluation method}

Subjective and quantitative evaluations were carried out by creating the actual pathological images of the target 


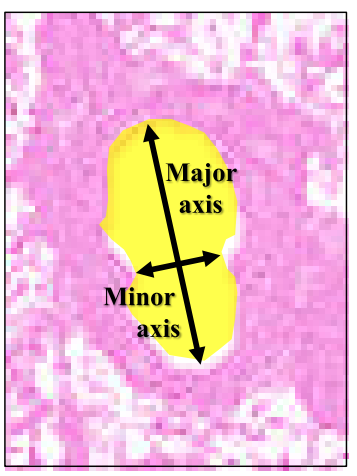

(a) Pseudo-histological image (lung)

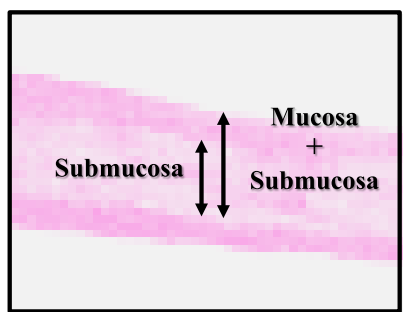

(c) Pseudo-histological image (colon)

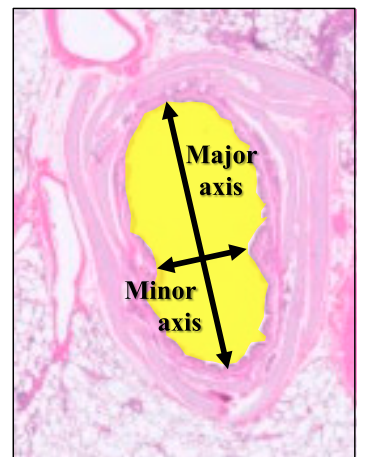

(b) Pathological image (lung)

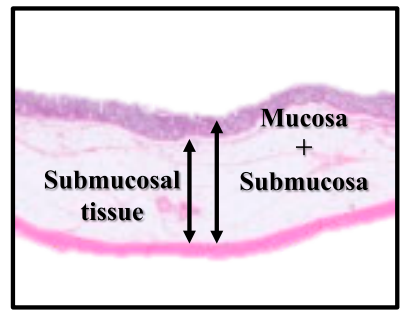

(d) Pathological image (colon)

Fig. 4. Quantitative evaluations of the lung and colon. (a) Pseudo-histological image (lung). (b) Pathological image (lung). (c) Pseudo-histological image (colon). (d) Pathological image (colon).

organ and comparing them with the pseudo-histological images obtained by the developed system. The experimental conditions are displayed in Table 1. A method for creating a pathological image and that for quantitative evaluation are described below.

\section{(1) Creating pathological images}

After the CT scan, the porcine organs were cut with a thickness of $5 \mathrm{~mm}$ using a scalpel (Fig. 3c), and fitted into an embedding cassette (Fig. 3d). Then, to make waterinsoluble paraffin penetrate into the tissue, the organs were dehydrated with a serial ethanol gradient. Subsequently, dealcoholization was carried out by placing them in a mediator (xylene), after which they were placed in a paraffin melter and allowed to penetrate. This was automatically carried out by the paraffin embedding system (manufactured by Sakura Finetek Japan, VIP-VII). Finally, the embedded cut-out section was immersed in paraffin, placed in a mold and solidified in a block shape under cooling to form a paraffin block.

The obtained paraffin block was sliced to a thickness of $3 \mu \mathrm{m}$ using a sliding microtome (ROM-380, Yamato Koki Co., Ltd.). The paraffin section was attached to a slide glass. Furthermore, the paraffin sections were stained based on the most basic tissue-staining method, the Hematoxylin and eosin (HE) staining method [5].

Pathological image data were obtained by scanning the glass specimen obtained through these processes using a scanner (GT-X980, manufactured by EPSON).

To create a pseudo-histological image with the same cross-section as the actual pathological image, we searched for the slice image that best matched the actual pathological image among the volume data obtained using our microCT system.

(2) Quantitative evaluation

The structures in the same cross-section of the pseudohistological image obtained using the developed system and the created pathological image were compared, and quantitative evaluation was performed for the lung and colon. In the lung specimen, we focused on bronchial tubes in the cross section. In these images, the lengths of the major and minor axes of the tube were measured. Using these two values, the aspect ratio was calculated (Fig. 4a, 4b). With regard to the colon, malignant tumors begin in the mucosal layer and invade submucosal tissues. In this study, we focused on the mucosal layer and the submucosal tissue layer. As shown in Fig. 4c, 4d, the ratio of the thickness of the submucosal tissue layer to the total thickness of the mucosal and submucosal tissue layers was calculated as the ratio of membrane layer. Then, quantitative evaluation was performed by comparing the ratios calculated for the pseudo-histological and pathological images of the same cross-section for each organ. In this study, we prepared five lung and colon slices for evaluation.

\section{II.5. Ethical approval}

All applicable international, national, and/or institutional guidelines for the care and use of animals were followed. Fujita Health University ethical committee for 
animal care approved the use of pig cadavers from a slaughterhouse without permission.

\section{Results}

\section{III.1. Visualization ability evaluation}

Fig. 5 shows the tomographic images of the lung and colon, obtained using image reconstruction via the developed system. In addition, Fig. 6 shows VR images of the lung and colon, provided by the developed software.

\section{III.2. Comparative evaluation}

Fig. 7 shows the results of the comparison of the pseudo-histological image and the pathological image obtained by the developed system. Table 2 shows the quantitative evaluation results for the lung and colon. The error rate was obtained by dividing the difference between the ratios corresponding to the pseudo-histological image and the pathological image by the ratio corresponding to the pathological image. The average error ratio of the aspect ratio between the two images for the lung was $9.95 \%$, and that of the layer thickness ratio between the two images for the colon was $5.42 \%$.

\section{Discussion}

Porcine lungs and colon were imaged using the developed system, and the reconstructed images and VR images were evaluated with regard to visualization ability. In the acquired slice image (Fig. 5), microstructures such as bronchial tubes, bronchioles, and interlobular walls of the

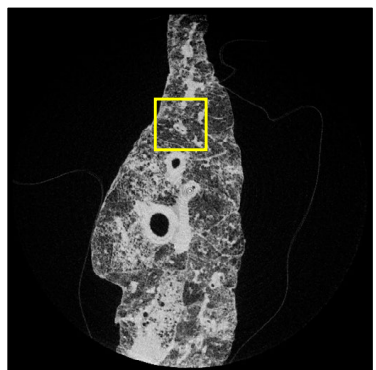

(a) CT slice image (lung)

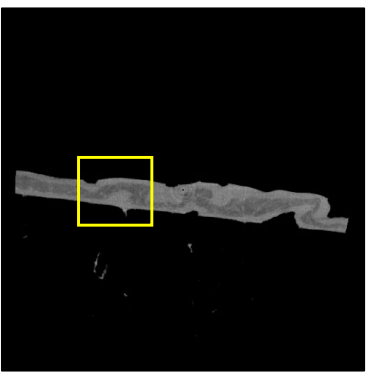

(c) CT slice image (colon)

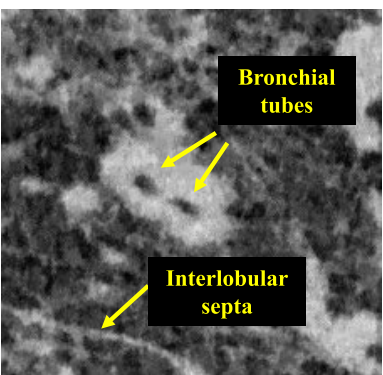

(b) Enlarged view (lung)

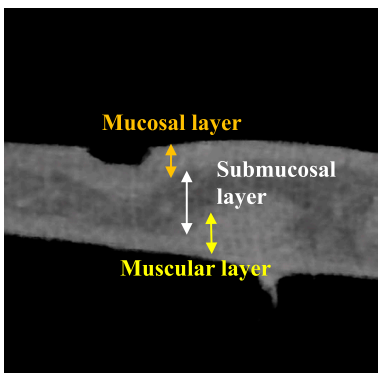

(d) Enlarged view (colon)
Fig. 5. Micro-CT images of the lung and colon. (a) CT slice image (lung). (b) Enlarged view (lung). (c) CT slice image (colon). (d) Enlarged view (colon)

lung were clearly observed. In the colon, a three-layer structure comprising a mucosal layer, submucosal tissue layer, and proper muscular layer was clearly observed. These results show that the developed system is capable of non-destructively acquiring high-resolution tomographic

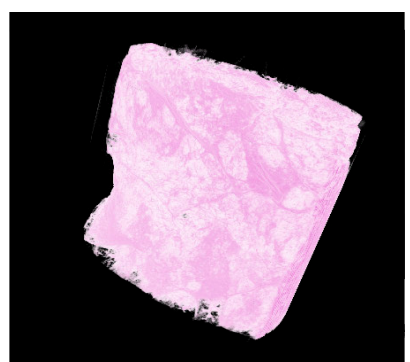

(a) Overall VR image (lung)

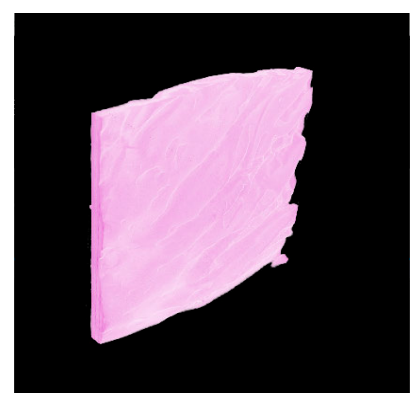

(c) Overall VR image (colon)

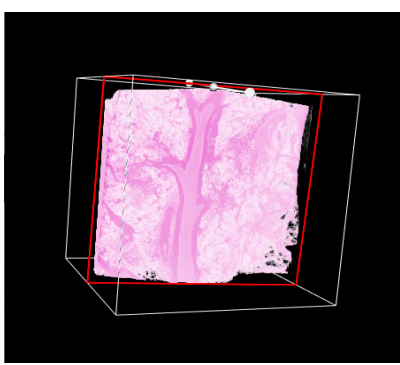

(b) Cross-sectional VR image (lung)

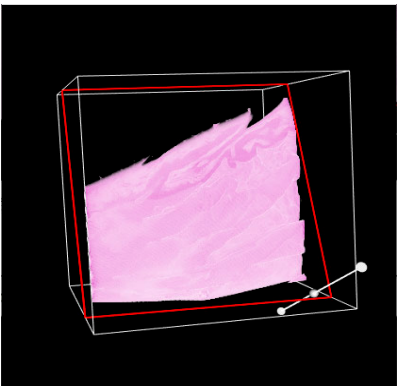

(d) Cross-sectional VR image (colon)

Fig. 6. Volume-rendered images of the lung and colon. (a) Overall VR image (lung). (b) Cross-sectional VR image (lung). (c) Overall VR image (colon). (d) Cross-sectional VR image (colon). 


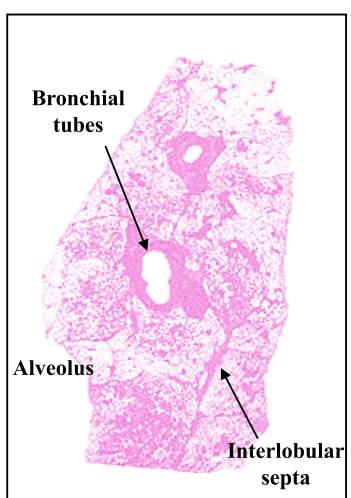

(a) Pseudo-histological image (lung)

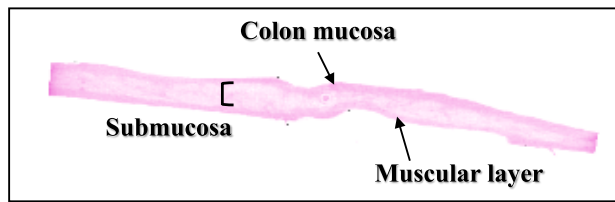

(c)Pseudo-histological image (colon)

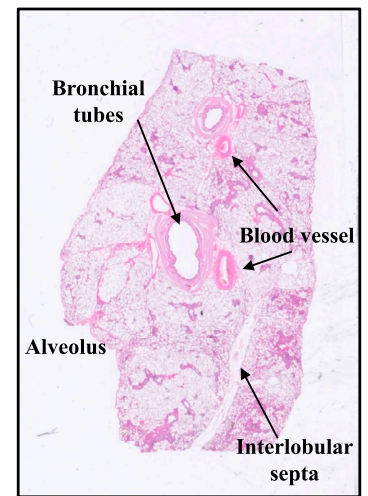

(b) Actual pathological image (lung)

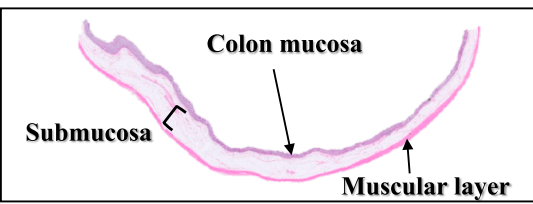

(d) Actual pathological image (colon)

Fig. 7. Comparative results for the lung and colon. (a) Pseudo-histological image (lung). (b) Actual pathological image (lung). (c) Pseudo-histological image (colon). (d) Actual pathological image (colon).

Table 2. Quantitative evaluation results

(a) Aspect ratio in lung specimen

\begin{tabular}{|c|c|c|c|}
\hline & Aspe & tio & \\
\hline & $\begin{array}{l}\text { Pseudo-histological } \\
\text { image (major axis }{ }_{[\mathrm{mm}]} / \\
\left.\text { minor axis }_{[\mathrm{mm}]}\right)\end{array}$ & $\begin{array}{c}\text { Pathological image } \\
\text { (major axis } \\
\left.\text { minor axis }_{[\mathrm{mm}]}\right)\end{array}$ & $\begin{array}{c}\text { Error rate } \\
{[\%]}\end{array}$ \\
\hline No. 1 & $1.80(3.99 / 2.22)$ & $2.07(3.95 / 1.90)$ & 13.0 \\
\hline No. 2 & $2.07(4.02 / 1.94)$ & $2.18(4.30 / 1.97)$ & 4.13 \\
\hline No. 3 & $2.24(4.12 / 1.84)$ & $2.57(4.24 / 1.65)$ & 12.8 \\
\hline No. 4 & $2.06(4.08 / 1.98)$ & $2.25(4.16 / 1.85)$ & 8.44 \\
\hline No. 5 & $1.55(3.82 / 2.46)$ & $1.75(3.55 / 2.03)$ & 11.4 \\
\hline
\end{tabular}

(b) Ratio of membrane layer in colon specimen

\begin{tabular}{lccc}
\hline & \multicolumn{2}{c}{ Ratio of membrane layer } & Error rate \\
\cline { 2 - 3 } & $\begin{array}{c}\text { Pseudo-histological image } \\
\left(\mathrm{a}_{[\mathrm{mm}]} /(\mathrm{a}+\mathrm{b})_{[\mathrm{mm}]}\right)\end{array}$ & $\begin{array}{c}\text { Pathological image } \\
\left(\mathrm{a}_{[\mathrm{mm}]} /(\mathrm{a}+\mathrm{b})_{[\mathrm{mm}]}\right)\end{array}$ & \\
\hline No. 1 & $0.77(2.01 / 2.59)$ & $0.86(1.59 / 1.86)$ & 10.5 \\
No. 2 & $0.81(1.65 / 2.03)$ & $0.80(1.75 / 2.17)$ & 1.25 \\
No. 3 & $0.67(1.55 / 2.30)$ & $0.73(1.09 / 1.50)$ & 8.22 \\
No. 4 & $0.80(1.92 / 2.40)$ & $0.84(1.63 / 1.95)$ & 4.76 \\
No. 5 & $0.82(1.86 / 2.26)$ & $0.84(1.74 / 2.06)$ & 2.38 \\
\hline
\end{tabular}

a: Submucosal tissue thickness, b: Mucosal layer thickness.

images of organs (lung, colon). However, a slight blur was observed in the tomographic image. This can be attributed to the deformation and movement of the subject during rotation upon the collection of projection data. To reduce blur, it is necessary to improve the fixture and optimize the rotation speed. Next, owing to the VR images of the lung and colon (Fig. 6), the entire organ in the whole image can be three-dimensionally indicated, and the internal structure can be confirmed from the cutting cross-sectional image. Therefore, by scanning and analyzing the sample before cutting, it is possible to repeatedly observe any cross-section in a non-destructive manner, which will support accurate determination of the cutting position.

A comparative evaluation of the pseudo-histological image and the pathological image obtained using the cutting support software in this system was performed. In the evaluation of the lung tissue, shown in Fig. 7, the network structure of the alveoli and the interlobular septa observed in the pathological image were observed in the pseudohistological image. However, in the pseudo-histological image, a portion corresponding to a cavity in the bronchus could not be observed. This may be because formalin with a high CT value remained in the bronchial tubes. Regarding the colon, the three-layer structure observed in the pathological image was clearly observed in the pseudohistological image, as shown in Fig. 7c and 7d. However, a change in outline shape between the pseudo-histological and pathological images was observed. It is considered that this is due to heating and stretching in the process of embedding and slicing when preparing a glass specimen. In addition, as the difference in pixel value between the mucosal layer and the serosal layer in the pathological image could not be determined, it was difficult to reproduce the difference in staining between the mucosal layer and the proper muscular layer. This is because the difference in tissue density between the mucosal layer and the serosal layer was small, and it was difficult to reproduce the difference between the two layers using this system. With regard to 
the quantitative evaluation, the size of the bronchi in the lungs and the thickness of the membranes in the colon were assessed. Paraffin-embedded blocks were prepared to prepare pathological specimens, and the absolute dimensions varied depending on the handling method. Therefore, we evaluated the reproducibility based on the ratio of the size, the average error rate for each organ was $10 \%$ or less. These results indicate that the developed system exhibits high reproducibility.

To prepare a pathological specimen, a rough section of the organ was made, followed by paraffin embedding, thin sectioning, and staining. If the optimal cutout position can be determined using the micro-CT proposed in this study, it will lead to the creation of a specimen that can determine the correct size and extent of the lesion.

Future studies should consider evaluating the reproducibility by performing quantitative evaluations on samples, including lesions. Additionally, we should evaluate this system using human organs to introduce this system in clinical practice. In addition, although contrast-enhanced CT imaging was not used in this study, it is desirable to develop a rapid contrast-enhancing technique and investigate how it can increase tissue contrast.

In this study, we have developed a novel system to support the determination of the cutting position using micro-CT. This system consists of a dedicated micro-CT device that scans a specimen and obtains a CT image, in addition to a cutting support software for analysis. Evaluation of the visualization ability revealed it was possible to visualize the internal microstructure of the organs. In addition, the comparison results revealed that the pseudohistological image and the pathological image had a high degree of coincidence. These findings imply that it is possible to observe the internal structure of the pathological material repeatedly and non-destructively by using the proposed system before cutting the pathological material. Therefore, the developed system is useful for pathological diagnosis.

\section{Conflicts of Interest}

The authors declare that they have no conflict of interest.

\section{Acknowledgement}

We are grateful to Mr. Kazuya Shiogama of Fujita Health University for creating the glass specimen.

\section{References}

1. Avila, L. S. (2010) The VTK User's Guide, 11th ed., Kitware,
New York, pp. 25-29.

2. Badea, C. T., Drangova, M., Holdsworth, D. W. and Johnson, G. A. (2008) In vivo small-animal imaging using micro-CT and digital subtraction angiography. Phys. Med. Biol. 53; R319-R350.

3. Buie, H. R., Campbell, G. M., Klinck, R. J., MacNeil, J. A. and Boyd, S. K. (2007) Automatic segmentation of cortical and trabecular compartments based on a dual threshold technique for in vivo micro-CT bone analysis. Bone 41; 505-515.

4. Feldkamp, L. A., Davis, L. C. and Kress, J. W. (1984) Practical cone-beam algorithm. J. Opt. Soc. Am. 1; 612-619.

5. Fischer, A. H., Jacobson, K. A., Rose, J. and Zeller, R. (2008) Hematoxylin and eosin staining of tissue and Cell Sections. CSH Protoc. 2008; pdb.prot4986.

6. Holdsworth, D. W. and Thornton, M. M. (2002) Micro-CT in small animal and specimen imaging. Trends Biotech. 20; 34-39.

7. Kawata, S. and Minami, S. (1986) The principle and applications of optical microscope tomography. Acta Histochem. Cytochem. $19 ; 73-81$.

8. Matsubara, O. and Maruyama, T. (2009) Clinical Laboratory Course: Pathology/Pathological Examination. Medical and Dentistry Co, Tokyo, pp. 235-238.

9. Paulus, M. J., Geason, S. S., Kennel, S. J., Hunsicker, P. R. and Johnson, D. K. (2000) High resolution X-ray tomography: an emerging tool for small animal cancer research. Neoplasia 2; 36-45.

10. Ritman, E. L. (2011) Current status of developments and applications of micro-CT. Annu. Rev. Biomed. Eng. 15; 531-552.

11. Ruhli, F., Kuhn, G., Evison, R., Muller, R. and Schultz, M. (2007) Diagnostic value of micro-CT in comparison with histology in the qualitative assessment of historical human skull bone pathologies. Am. J. Phys. Anthropol. 133; 1099-1111.

12. Sarraj, W. M., Tang, R., Najjar, A. L., Griffin, M., Bui, A. H., Zambeli-Ljepovic, A., et al. (2015) Prediction of primary breast cancer size and T-stage using micro-computed tomography in lumpectomy specimens. J. Pathol. Inform. 6; 60-81.

13. Senter-Zapata, M., Patel, K., Bautista, P. A., Griffin, M., Michaelson, J. and Yagi, Y. (2016) The role of micro-CT in 3D histology imaging. Pathobiology 83; 140-147.

14. Shepp, L. A. and Kruskal, J. B. (1978) The new medical X-ray technology. Am. Math. Mon. 85; 420-439.

15. Tang, R., Buckley, J. M., Fernandez, L., Coopey, S., Aftreth, O., Michaelson, J., et al. (2013) Micro-computed tomography (Micro-CT): a novel approach for intraoperative breast cancer specimen imaging. Breast Cancer Res. Treat. 139; 311-316.

16. Tsutsumi, H. (2012) Quick Master Pathology. Scio Publishers Inc., Tokyo, pp. 156-163.

17. Watz, H., Breithecker, A., Rau, W. S. and Kriete, A. (2005) Micro-CT of the human lung: imaging of alveoli and virtual endoscopy of an alveolar duct in a normal lung and in a lung with centrilobular emphysema-Initial observations. Radiology 236; 1053-1058.

This is an open access article distributed under the Creative Commons License (CC-BY-NC), which permits use, distribution and reproduction of the articles in any medium provided that the original work is properly cited and is not used for commercial purposes. 